\title{
Validation of a simple analytical method by UV-Spectroscopy, for the quantification of metformin in tablets, applied to dissolution profiles.
}

\author{
Martínez-Reséndiz Jorge Ignacio, Bautista-Sánchez Urias, Chehue-Romero Alejandro, Bautista-Ruíz \\ Alejandra, Téllez-López Ana María and Robles-Piedras Ana Luisa * \\ Área Académica de Farmacia. Instituto de Ciencias de la Salud Universidad. Autónoma del Estado de Hidalgo. Circuito Ex- \\ Hacienda La Concepción, Km. 1.5 San Agustín Tlaxiaca, Hidalgo. c.p. 42160 México.
}

Publication history: Received on 13 March 2020; revised on 21 March 2020; accepted on 22 March 2020

Article DOI: https://doi.org/10.30574/gscbps.2020.10.3.0065

\begin{abstract}
In Mexico, Metformin (MTF) is an oral hypoglycemic drug that is part of the group of biguanides used as a first-line treatment for the management of Type 2 Diabetes Mellitus. In the Mexican pharmaceutical market, there is a wide variety of medicines containing this drug, hence the importance of evaluating the quality of the medicines sold in the country. One of the important chemical tests performed both in the drug development phase, and in the quality evaluation criteria during its production, is the dissolution test. This is the test where it simulates in vitro, the time it takes for a given drug to pass into its soluble form, thus establishing its release time. The dissolution test to assess bioavailability in vitro has already been included in several official regulations, as a quality control method that allows predicting the behavior of the drug in the pharmaceutical form after its administration. The aim of this work was to validate a simple and reliable analytical method to quantify MTF in immediate-release tablets by UV spectroscopy, for a comparative study of in vitro dissolution profiles. In dissolution medium of $0.68 \%$ potassium monobasic phosphate at $\mathrm{pH}$ 6.8, adjusted with sodium hydroxide, the method demonstrated linearity in the range of 2 to $16 \mu \mathrm{g} / \mathrm{L}$. The parameters evaluated complied with the provisions of the national and international guidelines for the validation of analytical methods; therefore, the analytical method meets the requirements for the quantification of Metformin in dissolution studies.
\end{abstract}

Keywords: Metformin; Simple spectroscopic method; Dissolution studies

\section{Introduction}

In Mexico, Metformin (MTF) is an oral hypoglycemic drug that is part of the group of biguanides used as a first-line treatment for the management of Type 2 Diabetes Mellitus. In the Mexican pharmaceutical market, there is a wide variety of medicines containing this drug, hence the importance of evaluating the quality of the medicines sold in the country [1].

One of the important chemical tests performed both in the drug development phase, and in the quality evaluation criteria during its production, is the dissolution test. This is the test where it simulates in vitro, the time it takes for a given drug to pass into its soluble form, thus establishing its release time.

One of the needs of carrying out this test is the fact that it is possible to correlate the behavior of the drug in vivo, and its bioavailability can be inferred. Due to the direct relationship that exists between bioavailability and dissolution rate, a correlation that has been widely demonstrated, it has been established that the pharmaceutical form that rapidly

\footnotetext{
* Corresponding author: Robles-Piedras Ana Luisa
}

Copyright (C) 2020 Author(s) retain the copyright of this article. This article is published under the terms of the Creative Commons Attribution Liscense 4.0. 
releases your drug to the dissolution medium will have a better chance of being absorbed in better conditions with regarding that which releases it slowly or incompletely [2].

This explains the reason why dissolution tests have been included in several official regulations, such as a test to evaluate the bioavailability of an in vitro drug, applied as a quality control method that allows predicting the behavior of the drug in the pharmaceutical form after administration [3].

Therefore, the dissolution of a drug from its pharmaceutical form constitutes a critical step to evaluate and demonstrate that it meets the characteristics of quality, safety and efficacy. On the other hand, the development of dissolution methods may involve adapting an existing method by making minor changes or it is possible to obtain the optimal conditions from an experiment design.

In Mexico, dissolution methods must be validated, based on the requirements required by NOM-177-SSA-2013 and the current Pharmacopoeia, since the validation of the analytical methods is the confirmation, through objective evidence, of that the requirements for a specific intended use or application have been met. The validation of the method allows to have reliable analytical results and suitable for its proper use, so it must be broad enough to meet the application requirements for which it was developed $[3,4]$.

The aim of this work was to validate a simple and reliable analytical method to quantify MTF in immediate-release tablets by UV spectroscopy, for a comparative study of in vitro dissolution profiles.

\section{Material and methods}

\subsection{Instrumentation and analytical conditions}

The UV method was performed on a Single-beam VWR UV-1600PC UV-Visible spectrophotometer, with spectral bandwidth of $4 \mathrm{~nm}$, wavelength accuracy $\pm 0.5 \mathrm{~nm}$ and a pair of $1-\mathrm{cm}$ matched quartz cells was used to measure absorbance of solution. Working wavelength for UV method was 232nm of MTF.

\subsection{Solutions}

A $0.68 \%$ potassium monobasic phosphate solution was prepared at $\mathrm{pH} 6.8$, adjusted with sodium hydroxide ( $1 \mathrm{~N})$, which constituted the dissolution medium. With this medium, a stock solution of MTF was prepared at a concentration of 100 $\mu \mathrm{g} / \mathrm{mL}$.

\subsection{Validation of the analytical method}

The validation of the analytical method was carried out by determining the parameters of suitability and linearity of the system, linearity of the method, precision and accuracy, limit of detection and quantification and stability of the sample, at a wavelength of $232 \mathrm{~nm}$. MTF reference solutions and samples for each performance parameter were prepared each day of analysis.

\subsubsection{Suitability}

Six consecutive readings of a $10 \mu \mathrm{l} / \mathrm{mL}$ MTF solution were made. The system is considered stable when the coefficient of variation of the response $(\% \mathrm{CV})$ is less than $2 \%$.

\subsubsection{System linearity}

From a stock solution, 3 calibration curves were prepared. Aliquots were extracted and taken to a $25 \mathrm{~mL}$ volumetric flask with $0.68 \%$ potassium monobasic phosphate, to obtain concentrations of $2,4,6,8,10,12$ and $16 \mu \mathrm{g} / \mathrm{mL}$. The analytical response was measured at $232 \mathrm{~nm}$. The value of slope $b_{1}$, the ordinate at origin $b_{0}$, the coefficient of determination $r^{2}$ and the confidence interval for slope IC $\left(\beta_{1}\right)$ were calculated. Acceptance criteria: $r^{2}$ must be greater than 0.98; IC $\left(\beta_{1}\right)$ must not include zero.

\subsubsection{Linearity of the method}

From a stock solution, 3 calibration curves were prepared. Aliquots were extracted and taken to a $25 \mathrm{~mL}$ volumetric flask with $0.68 \%$ potassium monobasic phosphate, to obtain concentrations of $2,4,6,8,10,12$ and $16 \mu \mathrm{g} / \mathrm{mL}$. The analytical response of each was measured and the amount of MTF recovered was determined. The ratio of the amount added vs. the amount recovered was reported. The correlation coefficient (r), coefficient of determination $\left(\mathrm{r}^{2}\right)$, the 
confidence interval for the slope $\left[\operatorname{CI}\left(\beta_{1}\right)\right]$ and the ordinate at the origin $\left[\operatorname{CI}\left(\beta_{0}\right)\right]$, the coefficient of variation of the regression $\left(\mathrm{CV}_{\mathrm{y} / \mathrm{x}}\right)$ and the percentage of recovery were calculated, as well as the confidence interval of the arithmetic mean of the percentage of recovery [IC $(\mu)]$. Acceptance criteria: the value of $r$ must be $\geq 0.99$, the value of $r^{2}$ must be $\geq$ 0.98 , the value of $\operatorname{CI}\left(\beta_{1}\right)$ must include the unit, the value of $\operatorname{CI}\left(\beta_{0}\right)$ must include zero, the value of $\mathrm{CV}_{y / x}$ should be $\leq 3 \%$, the $\mathrm{CI}(\mu)$ should include $100 \%$ and the arithmetic mean of the recovery percentage should be in the range $98-102 \%$.

\subsubsection{Accuracy and Precision}

From the data obtained in the linearity of the method, the mean percentage recovery of MTF was calculated (it should not vary by more than $3 \%$ with respect to the nominal amount at each point). With the accuracy data of the method, the $\% \mathrm{CV}$ of the quantified percentage was calculated to determine repeatability. The $\% \mathrm{CV}$ of the percentage of the quantified drug must be less than or equal to $3 \%$.

\subsubsection{Reproducibility}

The effect of two analysts on two different days was evaluated, analyzing samples in triplicate and the recovery percentage was calculated.

\subsubsection{Limit of detection and Limit of quantification}

In this study, the limits of detection (LD) and quantification (LQ) were calculated based on the standard deviation of the response and the slope (see equation 1 and equation 2). From the calibration curves of the method, two parameters were taken: the slope $(\beta 1)$ and the standard deviation of the intercept or ordered to the origin $(S \beta 0)$.

$$
\text { Equation 1. } L D=\frac{3.3 s_{\beta_{0}}}{\beta_{1}} \quad \text { Equation 2. } L C=\frac{10 s_{\beta_{0}}}{\beta_{1}}
$$

\section{Results and discussion}

Table 1 shows the results of the system linearity assessment. According to the results obtained, the value of the slope, the ordinate at the origin, the coefficient of determination and the confidence interval for the slope, meet the acceptance criteria, obtaining a $\mathrm{r}^{2}$ greater than 0.98 and the $\mathrm{CI}\left(\beta_{1}\right)$ does not include zero. Table 2 shows the results of the statistical analysis that confirms the linearity of the method. Based on the results obtained from the measurement of the response of 7 levels of MTF concentration in phosphate solution, the statistical analysis showed that according to the established acceptance criteria, the method is linear, ensuring that the results obtained were proportional to the concentrations of the reference substance within the determined range. A coefficient of determination of 0.999 was obtained, the unit is included in the confidence interval for the population mean, the value of zero is within the confidence interval for the ordinate at the origin and the coefficient of variation of the regression is less than $2 \%$ ( $C V=1.2)$.

Table 1 Statistical analysis to determine the linearity of the system

\begin{tabular}{lllll}
\hline $\begin{array}{l}\text { MTF } \\
\begin{array}{l}\text { Concentration } \\
(\boldsymbol{\mu g} / \mathbf{m L})\end{array}\end{array}$ & $\begin{array}{l}\text { Absorbance } \\
\mathbf{1}\end{array}$ & $\begin{array}{l}\text { Absorbance } \\
\mathbf{2}\end{array}$ & $\begin{array}{l}\text { Absorbance } \\
\mathbf{3}\end{array}$ & $\begin{array}{l}\text { Mean } \\
\boldsymbol{\mu}\end{array}$ \\
\hline 2 & & & & \\
4 & 0.258 & 0.212 & 0.234 & 0.235 \\
6 & 0.430 & 0.409 & 0.392 & 0.410 \\
8 & 0.653 & 0.620 & 0.640 & 0.638 \\
10 & 0.876 & 0.865 & 0.868 & 0.870 \\
12 & 1.090 & 1.136 & 1.059 & 1.095 \\
16 & 1.260 & 1.253 & 1.246 & 1.253 \\
& 1.678 & 1.632 & 1.676 & 1.662 \\
& & $\mathrm{r}$ & $\mathrm{r}^{2}$ & $\mathrm{IC}\left(\beta_{1}\right)^{\mathrm{a}}$ \\
\hline
\end{tabular}

a Confidence interval for the slope 
Table 2 Statistical analysis to determine the linearity of the method

\begin{tabular}{|c|c|c|c|c|c|}
\hline Mean & $S$ & $\mathrm{CV} \%{ }^{\mathrm{a}}$ & $C V_{y / x}(\%)^{b}$ & $\mathbf{r}$ & $\mathbf{r}^{2}$ \\
\hline 100.855 & 1.281 & 1.270 & 1.029 & 0.999 & 0.998 \\
\hline$I^{\prime c}(\beta 0)+$ & $\mathrm{IC}^{\mathrm{c}}(\boldsymbol{\beta 0})-$ & $\operatorname{ICd}(\beta 1)+$ & $\operatorname{ICd}(\beta 1)-$ & $\mathrm{ICe}(\mu)+$ & $\operatorname{ICe}(\mu)-$ \\
\hline 1.069 & 0.981 & 101.880 & 99.829 & 101.438 & 99.387 \\
\hline e & $\begin{array}{r}\text { b Co } \\
{ }^{c} \text { Confid }\end{array}$ & $\begin{array}{l}\text { a Coefficier } \\
\text { ficient of vari } \\
\text { hce interval of } \\
\text { Confidence in } \\
\text { al of the arith }\end{array}$ & $\begin{array}{l}\text { of variation } \\
\text { tion of the regre } \\
\text { he ordinate at } t \\
\text { erval of the slop }\end{array}$ & $\begin{array}{l}\text { on } \\
\text { origin }\end{array}$ & \\
\hline
\end{tabular}

The determination of the parameters of accuracy and repeatability was carried out using the equation of the straight line obtained from the ratio of MTF concentration to absorbance, and the calculation of the quantity and percentage of drug recovered. With the statistical analysis performed, and in accordance with the acceptance criteria, in the evaluation of the parameters of accuracy and repeatability of the method, a CV of 1.65\% was obtained. Similarly, an interval was obtained that includes $100 \%$ and an arithmetic mean of the recovery percentage of $100.4 \%$; therefore it was shown that the method is accurate and repeatable (Table 3).

Table 3 Statistical analysis to evaluate the repeatability of the method

\begin{tabular}{|c|c|c|c|c|}
\hline Curve & MTF Concentration $(\mu \mathrm{g} / \mathrm{mL})$ & Absorbance & Amount recovered & $\%$ Amount recovered \\
\hline \multirow{7}{*}{1} & 2 & 0.252 & 2.039 & 101.961 \\
\hline & 4 & 0.445 & 3.925 & 98.137 \\
\hline & 6 & 0.647 & 5.911 & 98.513 \\
\hline & 8 & 0.872 & 8.113 & 101.409 \\
\hline & 10 & 1.093 & 10.276 & 102.765 \\
\hline & 12 & 1.300 & 12.31 & 102.582 \\
\hline & 16 & 1.655 & 15.789 & 98.683 \\
\hline \multirow{7}{*}{2} & 2 & 0.250 & 2.016 & 100.784 \\
\hline & 4 & 0.449 & 3.967 & 99.167 \\
\hline & 6 & 0.669 & 6.124 & 102.059 \\
\hline & 8 & 0.839 & 7.787 & 97.341 \\
\hline & 10 & 1.072 & 10.07 & 100.696 \\
\hline & 12 & 1.293 & 12.243 & 102.026 \\
\hline & 16 & 1.674 & 15.975 & 99.847 \\
\hline \multirow{7}{*}{3} & 2 & 0.253 & 2.045 & 102.255 \\
\hline & 4 & 0.458 & 4.055 & 101.373 \\
\hline & 6 & 0.664 & 6.075 & 101.242 \\
\hline & 8 & 0.854 & 7.939 & 99.240 \\
\hline & 10 & 1.053 & 9.883 & 98.833 \\
\hline & 12 & 1.283 & 12.145 & 101.209 \\
\hline & 16 & 1.653 & 15.767 & 98.542 \\
\hline$r_{\text {amount recovered }}$ & 0.999 & & & \\
\hline \multirow[t]{2}{*}{$\mathrm{r}^{2}$ amount recovered } & 0.998 & & & \\
\hline & Mean \% & 100.413 & & \\
\hline \multirow[t]{2}{*}{$y=0.102 x+0.0444$} & Sa $\%$ & 1.662 & & \\
\hline & $\mathrm{CV}$ b \% & 1.655 & & \\
\hline
\end{tabular}

The reproducibility of the method was carried out by applying two random events (analyst, day) were evaluated, analyzing a concentration level $(10 \mu \mathrm{g} / \mathrm{mL})$ in triplicate to test each condition. Table 4 shows the arithmetic mean, standard deviation (S) and\% CV calculated from the absorbance recorded of the sample analyzed by 2 different analysts on 2 different days with the same instruments and equipment. According to the acceptance criteria, a CV of $1.36 \%$ was obtained. Therefore, it was shown that the method is reproducible. 


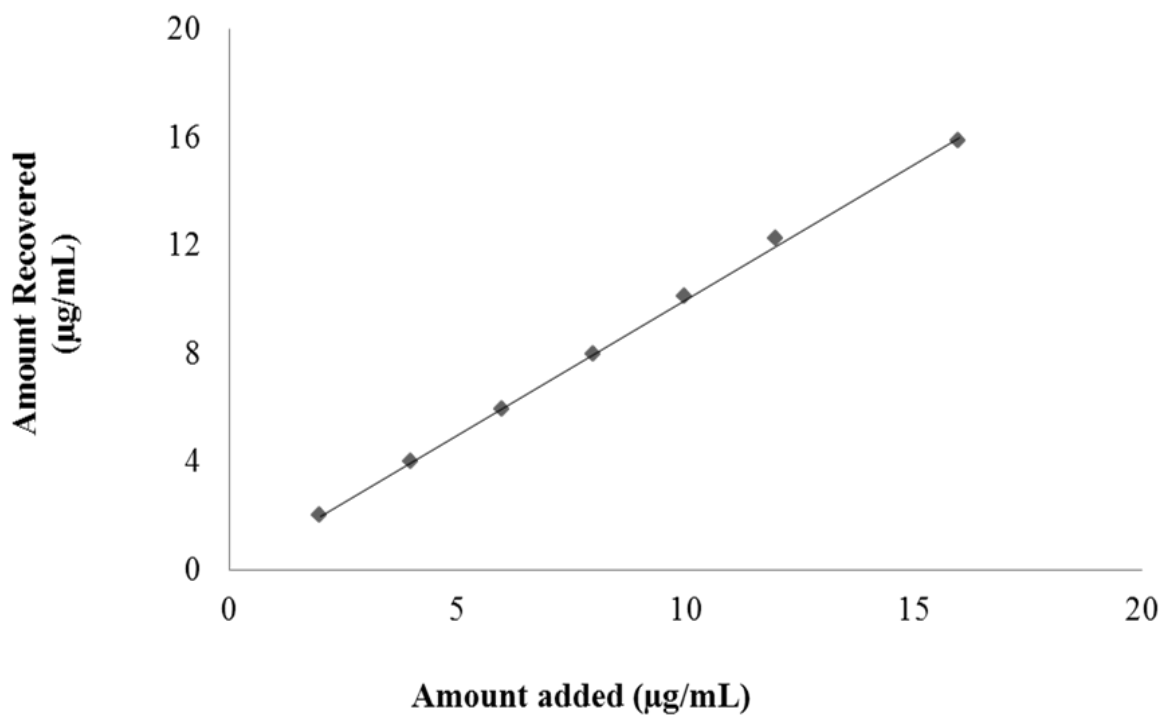

Figure 1 Relationship between the theoretical amounts of MTF added vs. amount recovered

Table 4 Statistical analysis to determine reproducibility of the method

\begin{tabular}{lll}
\hline MTF Concentration $\mathbf{1 0} \boldsymbol{\mu g} / \mathbf{m L}$ & Day $\mathbf{1}$ Absorbance & Day 2 Absorbance \\
\hline \multirow{2}{*}{ Analyst 1 } & 1.093 & 1.062 \\
& 1.072 & 1.081 \\
Mean & 1.053 & 1.054 \\
& 1.073 & 1.066 \\
Analyst 2 & 1.062 & 1.075 \\
& 1.081 & 1.094 \\
Mean & 1.054 & 1.077 \\
Sa & 1.066 & 1.082 \\
Global mean & 0.015 & \\
\%CVb & 1.069 & \\
\hline \multicolumn{2}{c}{ a Standard deviation } \\
\end{tabular}

The limit of quantification and limit of detection was calculated according to equation 1 and equation 2, from the calibration curve of the method, two parameters were taken: the slope $(\beta 1)$ and the standard deviation of the intercept (S $\beta 0$ ), obtaining an LD of $0.028 \mu \mathrm{g} / \mathrm{mL}$ and an LC of $0.871 \mu \mathrm{g} / \mathrm{mL}$ (Table 5).

Table 5 Limit of Detection and Limit of Quantification of the method

\begin{tabular}{lcccl}
\hline $\mathbf{S}$ & $(\boldsymbol{\beta 1})^{\mathbf{a}}$ & $(\mathbf{S} \boldsymbol{\beta} \mathbf{0})^{\mathbf{b}}$ & $\mathbf{L D}(\boldsymbol{\mu g} / \mathbf{m L})$ & $\mathbf{L C}(\boldsymbol{\mu g} / \mathbf{m L})$ \\
\hline 0.2 & 0.102 & 0.044 & 0.028 & 0.871 \\
\hline \multicolumn{5}{c}{$\begin{array}{c}\text { a Slope } \\
\text { b Standard deviation of the intercept }\end{array}$}
\end{tabular}




\section{Conclusion}

The validated method for quantifying MTF in a $0.68 \%$ potassium monobasic phosphate solution at $\mathrm{pH} 6.8$, adjusted with sodium hydroxide $(1 \mathrm{~N})$ in a concentration range of 2 to $16 \mathrm{mg} / \mathrm{mL}$ proved to be linear, accurate, precise and reliable; so, the method is apt to be used to quantify MTF in dissolution studies.

\section{Compliance with ethical standards}

\section{Acknowledgments}

The authors would like to appreciate the technical staff, Laboratory of Biopharmaceutics, University of Hidalgo, for their kind assistance.

\section{Disclosure of conflict of interest}

The authors declare that there is no conflict of interest.

\section{References}

[1] Markowicz-Piasecka M, Huttunen KM, Mateusiak L, Mikiciuk-Olasik E and Sikora J. (2017). Is Metformin a Perfect Drug? Updates in Pharmacokinetics and Pharmacodynamics. Current Pharmaceutical Design, 23(17), 25322550.

[2] Jambhekar SS and Breen PJ. (2013). Drug dissolution: significance of physicochemical properties and physiological conditions. Drug discovery Today, 18(23-24), 1173-1184.

[3] "Norma que establece las pruebas y procedimientos para demostrar que un medicamento es intercambiable". Norma Oficial Mexicana NOM-177-SSA1-2013. Diario Oficial de la Federación, 20 de septiembre de 2013.

[4] Farmacopea de los Estados Unidos Mexicanos. (2009). Comisión Permanente de Farmacopea de los Estados Unidos Mexicanos. 9ª edición. Secretaria de Salud. México, 2061.

\section{How to cite this article}

Martínez-Reséndiz JI, Bautista-Sánchez U, Chehue-Romero A, Bautista-Ruíz A, Téllez-López AM and Robles-Piedras AL. (2020). Validation of a simple analytical method by UV-Spectroscopy, for the quantification of metformin in tablets, applied to dissolution profiles. GSC Biological and Pharmaceutical Sciences, 10(3), 104-109. 